BIOFILMS

\title{
Clutch control
}

Biofilms have long been known to comprise architecturally complex communities of sessile bacterial cells, but what switches off bacterial motility within biofilms has been a mystery. Now, a new study published in Science reveals that in Bacillus subtilis biofilms, motility is switched off through the activity of a protein that acts as a clutch and disables the flagellar motor.

In B. subtilis, motility and biofilm formation are oppositely regulated by the master regulator $\underline{\operatorname{SinR}}$. Previous work had shown that $\operatorname{Sin} R$ is a direct negative regulator of the eps operon, which is responsible for the production of the extracellular matrix that is essential for biofilm formation, but precisely why $\sin R$ mutants are non-motile was unknown.

Kris Blair and colleagues began by looking at flagellar distribution and function in $\sin R$ mutants using a fluorescently labelled flagellar filament protein. They found that in $\sin R$ mutants, flagella are present but non-functional. To investigate in more detail how $\operatorname{SinR}$ regulates flagellar function, Blair et al. looked for suppressor mutations that restored motility in $\sin R$ mutants. Of the 18 suppressors that were isolated, 9 mapped to the epsE gene, and introduction of wild-type epsE could complement the inhibition of motility.

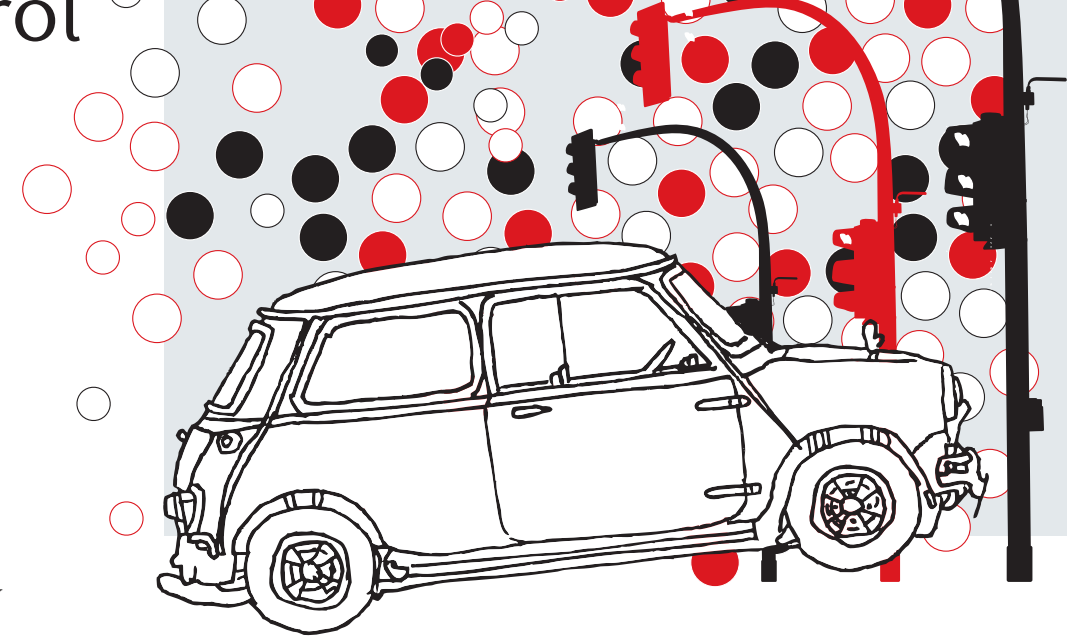

The authors therefore concluded that $\sin R$ mutants are non-motile because epsE is derepressed. Further work revealed that EpsE is sufficient for motility inhibition and that this inhibition involves stopping flagellar rotation.

So what is the target for EpsE? Again, the authors turned to suppressor mutations, but this time looked for suppressors that rescued motility in epsE mutants. All of the suppressors that were isolated mapped to $f l i G$, which encodes a component of the flagellar motor that is responsible for transmitting torque to the rotary motor through the MotA/B proton channel. The subcellular localization patterns of EpsE were studied in different mutant backgrounds using a fluorescent EpsE fusion protein, and the results confirmed that EpsE interacts with FliG in vivo and that this interaction is responsible for motility arrest. Finally, by tethering B. subtilis cells to a surface by a single flagellum and observing the rotation of the cell body in the presence and absence of EpsE, the authors discovered that EpsE acts as a clutch that disengages the flagellar rotor from the power source, the MotA/B proton channel.

So, the motility of $B$. subtilis cells within biofilms is shut down through the use of a molecular clutch, EpsE. The authors conclude that clutch control is a "simple, rapid and potentially reversible" way to switch off motility.

$$
\text { Sheilagh Molloy }
$$

ORIGINAL RESEARCH PAPER Blair, K. M. et al. A molecular clutch disables flagella in the Bacillus subtilis biofilm. Science 320, 1636-1638 (2008) 